\title{
Qnurealy

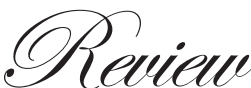 \\ A clinical primer of the role of gut microbiome in health and disease
}

\author{
Allison L. Yang ${ }^{1}$, Purna C. Kashyap ${ }^{2}$
}

ABSTRACT

Department of Internal Medicine ${ }^{1}$ and Gut microbiome represents the total microbes present in the gastrointestinal tract including Department of Gastroenterology and the genes they encode. These microbes primarily exist in a reciprocal state with the host Hepatology ${ }^{2}$,

Mayo Clinic, Rochester,

MN 55905 contributing several important functions such as carbohydrate fermentation, vitamin

Correspondence:

Dr. Purna C. Kashyap

Email: kashyap.purna@mayo.edu biosynthesis and regulation of the immune system. The gut microbiome represents a dynamic organ, which responds to changes in the host, such as genetics and age, as well as environment such as diet and antibiotics. While these microbes can adapt to change, any disturbance in this host-microbe equilibrium has the potential to initiate a cascade of events leading to a disease phenotype. In this review we highlight the emerging role of gut microbiome in different gastrointestinal and systemic diseases, the role of current therapies and development of future therapies targeting the gut microbiome as a potential mode of treatment.

KEYWORDS: Microbiota, Gastrointestinal, IBD, IBS, Malignancy, Obesity, Clostridium difficile associated disease, Probiotics, Prebiotics, Fecal Transplant

\section{Introduction}

The human gut microbiome is a dynamic array of microorganisms, including bacteria, Archaea, fungi and viruses that colonize the surfaces of the gastrointestinal (GI) tract. ${ }^{1}$ These organisms exist in precise immunological balance with the human host, which, despite being exquisitely sensitive to distinguishing self from other, allows their presence. The mutualistic equilibrium between host and microbiota has evolved over time into an expanding field of study, with increasingly important clinical implications for human health, pathogenesis of disease, and the future of medical treatments.

Advances in technology have allowed for the identification of previously non-culturable organisms, expanding our knowledge of the GI microbiota. It is now known that the average human has over 100 trillion microbes in the gut. ${ }^{2}$ These microbes form distinct communities depending upon their location and level of residence. ${ }^{2,3}$ Bacteria are the predominant member of the microbiota, and less than $0.1 \%$ are pathogenic.
Two phyla predominate: Bacteroidetes and Firmicutes, ${ }^{4}$ of which the majority are of the class Bacteroides and Clostridia. ${ }^{5}$ Proteobacteria is the third most common phyla, with Actinobacteria, Cyanobacteria, Fusobacteria, Spirochetes, and Verrucomicrobia detected at significantly lower densities. ${ }^{6}$ The majority of these microbes are anaerobic in that $>99 \%$ of bacteria isolated cannot grow in the presence of oxygen. Aside from bacteria, Methanobrevibactersmithii is the predominant archaeon in the GI tract. ${ }^{7}$

Recent knowledge of the healthy, human gut microbiome has come from 'The Human Microbiome Project', which analyzed stool samples from healthy individuals using $16 \mathrm{~S}$ rRNAmarker gene sequencing and metagenomic profiles generated by whole genome shotgun sequencing. ${ }^{8,9}$ Using the metagenomic profiles of 39 individuals, Arumugam et al. described three distinct groups or "enterotypes" based on gut microbial composition. Bacteroides was most abundant in 
enterotype 1, Prevotella in enterotype 2, and Ruminococcus in enterotype $3 .{ }^{10}$ Subsequent studies have identified gradients of Bacteroides and Prevotella in populations highlighting niche exclusivity as well as suggesting that enterogradients may be a more appropriate description than enterotypes.

The bacterial composition of the mucosa-associated microbiota varies longitudinally along the GI tract. The distal esophagus is dominated by Streptococcus species, with smaller contributions from Prevotella, Actinomyces, Lactobacillus and Staphylococcus. ${ }^{11}$ The stomach is also limited in microbial diversity, possibly due to the low $\mathrm{pH}$ environment. ${ }^{4}$ Helicobacter pylori accounts for the vast majority of the gastric microbiome and, in its absence, Streptococcus predominates. ${ }^{12}$ The microbiome of the human small intestine has not been as thoroughly investigated, although Streptococcus is known to be the predominant genus in the duodenum and jejunum. ${ }^{13}$ In the distal gut, the microbiome is enriched for fermentation of dietary fiber and processing of glycans to short-chain fatty acids. $^{14}$

The composition of the microbiome is influenced by several factors including age, gender, ethnicity, diet, hygiene, behavior, genetics, and co-morbid medical conditions. Age is particularly important and the gut microbiota composition may differ at different points within the life of the same individual. ${ }^{15}$ Colonization with gut microbiota begins at or just prior to birth, as the meconium of full-term neonates has been shown to contain bacteria. Vaginally-delivered infants have microbiomes that resemble the mothers' vaginal tract, whereas babies delivered by Caesarean section have microbiomes consisting largely of typical skin microbiota, including Staphylococcus, Corynebacterium and Propionibacterium. ${ }^{15-17}$ There is rapid development of the intestinal microbiome early in life and, by the age of 3 years, the microbiome composition of the GI tract is 40 to $60 \%$ that of an adult. The development of the microbiome peaks during adolescence and then stabilizes between the third and seventh decades of life. Beyond the seventh decade of life, the microbiome becomes comparatively less diverse with reduced stability, and proportions of Bifidobacteria, Faecalibacterium prausnitzii, and multiple members of Firmicutes decrease, whereas the numbers of E. coli, Proteobacteria and Staphylococcus increase. ${ }^{17}$

Long-term and short-term dietary intakes also play important roles. From infancy, human milk oligosaccharides, comprised of indigestible glycans, travel through the intestinal tract and support the growth of specific colonic bacteria such as Bifidobacterium, essentially functioning as prebiotics. ${ }^{18}$ The increased Bifidobacterium may strengthen the gut mucosa and protect against pathogens. ${ }^{19}$ More globally, diets that differ amongst cultures have also led to the development of different intestinal microbiomes. The most well-known study to demonstrate this effect was conducted by De Filippo et al and revealed vast differences in the composition of intestinal microbiota between children in rural Burkina Faso and children in more-developed Europe. ${ }^{20}$

The gut microbiota is a delicate balance of organisms that can be altered by innumerable factors, many of which have yet to be identified. Furthermore, the impact of these modifications on human health is not yet fully understood. However, as differences between the microbiota of the healthy and the sick have been identified, the microbiomehas become implicated in the development and progress of both GI diseases (infections, chronic diseases, malignancies) and extra-intestinal manifestations (obesity, neuropsychiatric conditions). This makes the microbiome an increasingly attractive target in the modulation of disease. As the role of the gut microbiota in the development of other medical conditions has been wellreviewed in the literature, this review will focus solely on the role of the gut microbiota in the pathogenesis of inflammatory bowel diseases (IBDs), functional bowel disorders, gastrointestinal infection with $C$. difficile, malignancies and metabolic disorders.

\section{Gut Microbiota in Inflammatory Bowel Disease}

The inflammatory bowel diseases (IBD) comprise Crohn's disease and ulcerative colitis (UC), and affect 3.6 million people worldwide. ${ }^{21-23}$ Both are relapsing inflammatory conditions affecting either the superficial layers of the colonic wall (UC) or the transmural GI tract (Crohn's). As with other chronic remitting and relapsing diseases, the etiology of IBD is multifactorial, with contributions from inheritable genetic components (163 host susceptibility loci identified to date), environmental factors, diet, and, more recently identified, the gut microbiota. ${ }^{24-26}$ The development of IBD in genetically susceptible individuals has been linked to an alteration in the composition of the microbiome subsequent to adysregulation of the mucosal immune system. The gut can be colonized with microbes that evade host immune detection and aberrantly activate a $\mathrm{T}$ cell-mediated immune response towards commensal gut microbiota. ${ }^{27}$

Metagenomic and 16S rRNA-based marker gene sequencing studies have demonstrated reductions in biodiversity in 
patients with IBD compared to healthy individuals. ${ }^{28} \mathrm{IBD}$-related changes include decreases in Firmicutes, Bacteroides, Clostridia, Ruminococcaceae, Bifidobacterium, F.prausnitzii, and Lactobacillus, the presence of Fusobacterium, and increases in Gammaproteobacteria and adherent-invasive $E$. coli. ${ }^{29,30}$ In fact, $E$. coli has been commonly isolated from ileal biopsy specimens and fecal samples of IBD patients. ${ }^{31}$ These adherent species invade epithelial cells and replicate within macrophages, inducing granuloma formation in vitro studies. ${ }^{32}$ Fusobacterium, comprised of gram-negative anaerobes that normally colonize the oral cavity, also exist in higher numbers in patients with IBD, and human isolates of Fusobacteriumvarium have induced the development of mucosal erosions in mice. ${ }^{33-35}$

The microbiota is essential in driving inflammation as well. Specific groups of bacterial species appear to be protective against IBD and their absence or limited presence may predispose to the development of colitis. The depletion of Faecalibacterium prausnitzii in IBD has been linked to decreases in anti-inflammatory cytokines, including interleukin 10 (IL-10), and higher risks of recurrent Crohn's disease postsurgery. ${ }^{36,37}$ Bifidobacterium and Lactobacillus down-regulate inflammatory cytokines and are protective; however, both are depleted in IBD. Similarly, Atarashi et al have shown that Bacteroides and Clostridium reduce intestinal inflammation by inducing the expansion of regulatory $\mathrm{T}$ cells, and the decreased presence of these bacteria is associated with increased mucosal inflammation. ${ }^{38}$ Lastly, Faecalibacterium, Phascolarctobacterium, and Roseburia play a major role in fermenting dietary fiber to yield the short-chain fatty acids acetate and butyrate, which, in turn, are implicated in inducing the expansion of regulatory $\mathrm{T}$ cells; the presence of these bacteria is reduced in patients with IBD. 2,30,39

Treatments targeting the microbiome can contribute significantly to the management of IBD. Prior to the development of current disease-modifying therapies, antibiotics were the mainstay of therapy. Enteric-coated antibiotics dramatically reduce intestinal inflammation and are still used for the treatment of pouchitis. ${ }^{40}$ However, antibiotics can alter both the gut microbial ecology and characteristics of the mucous layer, weakening its protective barrier functions and allowing for invasion by adherent bacterial species. Therefore, while effective, therapeutic alternatives to antibiotics continue to be investigated.

Though not well-studied in IBD, restoration of normal gut microbiota with a fecal microbiota transplant or the introduction of defined microbial communities may be potential therapies. Current data are limited by the paucity of controlled trials, with only a few case reports of successful fecal microbiota transplantation. Kelly et al reported improvement in symptoms within 4 months following fecal microbiota transplants in 6 patients with IBD. ${ }^{41}$ Kunde et al. reported a similar improvement in 7 out of 9 children with UC within 1 week following fecal transplantation. ${ }^{42}$ However, there are concerns about the introduction of bacteria into patients with already compromised immune systems and gut mucosal layers in the absence of well-controlled studies and a clear mechanism of therapeutic action.

In addition to microbiota transplantation, there have been various reports on the efficacy of prebiotics and probiotics. Increased dietary fiber may act as a beneficial prebiotic as it can be fermented to anti-inflammatory short-chain fatty acids. Prebiotic studies have been severely limited by small sample sizes but have shown that in Crohn's disease, fructooligosaccharides reduce pro-inflammatory interleukin 6 (IL-6) positive lamina propria dendritic cells; however, these reductions were not accompanied by significant changes in clinical status. ${ }^{43}$ Similarly, germinated barley foodstuffs, including Ispaghula husk, have been shown to have some effect on mild to moderate IBD, whereas inulin supplementation leads to increased butyrate and lower levels of Bacteroides fragilis and Bacteroidetes in the feces of patients with chronic pouchitis. ${ }^{44}$ Moreover, studies in migrant populations suggest individuals who move from Southeast Asia to western countries have a higher risk of developing IBD; this effect is likely secondary to changes in dietary habits and resultant effects on gut microbiota in genetically susceptible individuals. ${ }^{45}$.

Treatment with the probiotic BIFICO (Bifidobacterium, Lactobacillus, and Enterococcus oral capsules) increases Bacilli, Enterococci, Bifidobacteria, and Lactobacilli, decrease Bacteroides, ${ }^{46}$ and prevents UC flares. The probiotic VSL\#3 has demonstrated a positive effect in $\mathrm{UC}^{47}$ and has also been helpful in maintaining remission of pouchitis ${ }^{48-50}$ and Lactobacillus $G G$ helps with the maintenance of remission in UC patients with mild to moderate disease. However, randomized trials of patients with Crohn's disease failed to demonstrate that the use of Lactobacillus $G G$ as an adjuvant to standard therapy was effective in maintaining remission. ${ }^{51-}$ 53

In order to more effectively treat patients with IBD through manipulation of the gut microbiota, it will be necessary to move beyond a simple enumeration of the microbial content 
associated with these diseases and actually understand the role of gut bacteria in their pathogenesis. The declining cost of sequencing, enhanced bioinformatics methodology, improved capacity for handling big data and more effective disease modeling using gnotobiotic rodents should make this an achievable goal.

\section{Gut Microbiota in Irritable Bowel Syndrome}

Irritable bowel syndrome (IBS) is a widely prevalent chronic GI disorder affecting nearly 1 in 5 Americans.$^{54}$ Current criteria define IBS as abdominal discomfort or pain associated with two or more of the following at least $25 \%$ of the time: improvement with defecation, onset associated with a change in frequency of stool, or onset associated with a change in form (appearance) of stool. The main subtypes of IBS are based on the predominant bowel pattern and include constipationpredominant (IBS-C), diarrhea-predominant (IBS-D), and mixed subtypes. ${ }^{55}$

Several factors have been implicated in the sensorimotor dyfunction observed in IBS, with recent evidence suggesting that alterations in gut bacteria may play a key role. Inital crosssectional studies using 16S rRNA-based microbial community composition analyses revealed quantitative and qualitative changes to the gut microbiota. ${ }^{56}$ However, findings have been inconsistent likely due to heterogeneous study populations and differing methodologies for sample preparation and analysis. For example, using the traditional culture method, Balsari et al and Si et al showed decreased coliform bacteria, Bifidobacterium species, and Lactobacillus species in stool samples, whereas Matto et al, also using cultures, demonstrated an increase in coliform bacteria. ${ }^{57,58}$ Concurrently, Malinen et al have used qPCR to identify decreases in B. catenulatum, $\mathrm{Cl}$. Coccoides, and Lactobacillus and increased Veillonella species. ${ }^{59}$

A consistent finding among major studies of IBS has been decreased microbial diversity and temporal stability. Specifically, the gut microbiota has decreased diversity with losses in Bacteroidetes species, fecal Lactobacilli, Bifidobacteria and increases in Streptococci, E. coli, and Clostridium. ${ }^{60,61}$ Additionally, there is alteration in the anaerobe/aerobe ratio in patients with IBS, with some studies suggesting a decrease in anaerobes possibly due to an acceleration of transit in IBS-D. ${ }^{60,61}$

In addition to composition studies, studies have identified correlations between IBS symptoms and specific bacteria.
Emerging data suggest the gut microbiota may underlie the pathophysiology of IBS, as several peripheral mechanisms implicated in the disease, including visceral hyspersensitivity, altered intestinal barrier, modulation of the gut brain axis, can be influenced by gut microbiota. Lactobacilli and Bifidobacteria, often used for treating IBS are considered beneficial bacteria and inhibit the release of pro-inflammatory cytokines from dendritic cells; the absence or decreased presence of these bacteria can lead to increased cytokinemediated inflammation. ${ }^{62}$ Studies have also shown that patients with IBS have increased colonic mucosal expression of receptors for specific microbiota substances such as Toll-like receptor-4 (which has an affinity for bacterial lipopolysaccharides) ${ }^{63}$ and increased titers of circulating antibodies against components of indigenous microbiota, which lead to activation of the mucosal immune response and increased activated mast cells. ${ }^{56,64,65}$

As with other functional GI disorders, the gut microbiota has become an important target in the treatment of IBS, with therapy focused on correcting microbial imbalances. To this end, changes in diet, use of prebiotics and probiotics, and administration of antibiotics have all been employed. A low FODMAP (Fermentable Oligo- Di- Monosaccharides and Polyols) diet has been shown to reduce symptoms in patients with IBS; however, long-term benefit has not been demonstrated and there is concern regarding the potential loss of beneficial bacteria ${ }^{66}$ Prebiotics have proven more promising, with trans-galactooligosaccharide mixtures associated with reduced symptoms and the increased presence of beneficial bacteria such as Bifidobacteria. ${ }^{67}$

Several studies have indicated symptomatic improvement, including decreased symptoms of flatulence with administration of probiotics such as Bifidobacteria (B. infantis, B. lactis, B. bifidum), Lactobacillus acidophilus and Lactobacillus plantarum. ${ }^{68}$ Mixed preparations of probiotics have also led to decreased bowel movements, pain, and bloating. ${ }^{69}$ Lastly, while data are still limited, initial evidence is quite strong for the use of Bifidobacterium infantis for a 4week duration. Early anecdotal reports have also suggested a potential role for fecal material transplant in managing IBS symptoms. While the use of some antibiotics has been associated with the development of IBS, poorly-absorbed antimicrobials such as rifaximin may still be of benefit. ${ }^{70}$ The mechanism of action is largely unclear but rifaximin has been used successfully in several double-blind, placebo-controlled trials to improve symptoms of bloating and flatulence. However, 
one needs to exercise caution given the risk of developing resistant organisms.

As we systematically define the effect of gut microbiota on host function, we will likely find alterations in gut microbial function as a central consideration in IBS. The current generation of probiotics contains only mixtures of a few bacterial strains and has shown definite promise as a therapeutic agent. However they are limited due to lack of complexity and unclear mechanism of action. As we move ahead, the use of defined microbial communities aiming to restore host function will likely replace traditional probiotic approaches.

\section{Gut microbiota in Clostridium difficile infection}

Clostridium difficile ( $C$. difficile) is a gram positive, anaerobic, spore-forming bacterium and is the most common cause of hospital-acquired diarrhea. It affects more than 1 million patients in the United States each year, resulting in annual hospital costs of more than $\$ 3$ billion dollars. ${ }^{71,72}$ Watery diarrhea with lower abdominal pain and cramping are the cardinal clinical symptoms of this infection, and complications include toxic megacolon and severe systemic illness. $C$. difficile is highly prevalent in infants who typically remain asymptomatic and colonization decreases with age. ${ }^{73}$ Adults frequently exposed to health care are typically the only adult carriers of $C$. difficile, and these individuals are reservoirs for environmental contamination.

In contrast to other bacterial pathogens, previous antibiotic exposure appears to be a significant risk factor for the development of $C$ difficile colitis..$^{74,75}$ The highly diverse normal gut microbiota resists colonization by bacterial pathogens, but exposure to antibiotics among other factors can disrupt the normal gut microbial ecology with a decrease in overall biodiversity potentially allowing for colonization by an opportunistic pathogen such as $C$ difficile. This has led to use of antibiotics to develop animal models, for example, clindamycin exposure in mice allowed for long-term, nonfatal colonization with $C$. difficile. ${ }^{76}$ Similarly, Chen et al developed a mouse model of $\mathrm{C}$. difficile infection by exposing mice to 5 antibiotics followed by a single dose of clindamycin, leading to the development of severe colitis. ${ }^{75}$ Epidemiology studies of patients with $C$. difficile infection have confirmed the role of antibiotics in its development. However, the relationship between specific antibiotic-induced perturbations in gut microbiota and a predisposition to a $C$. difficile infection remains to be elucidated.

While antibiotics are a major risk factor, $30 \%$ of patients in the community develop $C$. difficile infection without prior antibiotic use, suggesting that other host, environmental, or microbial factors may also be involved in creating the very specific environment necessary for colonization by this one pathogenic species. ${ }^{77}$ Initial data suggest that alterations to the diversity of the GI microbiome may be involved in the development of $C$. difficile infection. In mouse models, Wilson and Perini demonstrated that a more diverse microbiota competes for available resources with $C$. difficile pathogens, thus suppressing infection by one agent. ${ }^{78,79}$ Fecal samples from patients with $C$. difficile have decreased Lachnospiraeceae and increased Enterobacteriaceae, ${ }^{80}$ Furthermore, the intestinal microbiome of patients with recurrent $C$. difficile infection is also altered, with lower diversity and differences in composition as compared to patients who were successfully treated and eradicated of $C$. difficile. It is not clear though whether these changes contribute to symptomatic $C$. difficile infection or alternately result from C. difficile colonization. A significant gap in the field remains regarding specific antibiotic induced perturbations in gut microbiota, which in turn may predispose an individual to $C$. difficile infection. Moreover specific factors influencing gut microbiota in healthy individuals and factors predisposing them to $C$. difficile infection without prior antibiotic use also need to be clarified.

The mainstay of treatment for $C$. difficile colitis has been cessation of the culprit antibiotic and initiation of treatment with other antimicrobial agents, typically oral metronidazole, vancomycin ${ }^{81}$ orfidaxomicinor rifaximin..$^{82}$ Probiotics have had limited success but show some protection against the development of antibiotic-associated diarrhea. Lactobacillus rhamnosus and $S$. boulardii are the two most commonly studied strains, but limitations to these studies have included small sample sizes and discrepancies among trial methodologies. ${ }^{83}$

It has been well delineated that the gut microbiota compositions between patients infected with $C$. difficile and those without this pathogenic species are clearly different. Modification of the microbiota through fecal microbiota transplantation is thus an attractive therapeutic intervention and has been shown to cure recurrent infections in up to $95 \%$ of patients. ${ }^{71}$ Fecal microbiota transplantation can be performed by two methodologies. The first involves transplantation of an entire microbial community from donor stool. ${ }^{84,85}$ 
Longitudinal studies following transplantation have shown that use of donor feces quickly leads to donor-like composition of the gut microbiota in recipients. ${ }^{86,87}$ More recently, the direction of research has focused on a second methodology involving the administration of controlled mixtures of microbes extracted from stool and composed in laboratory-based settings. This new therapeutic option was developed not only in response to aesthetic concerns but also to make treating $C$. difficile with transplanted fecal material a more clinical, pharmacologic strategy. To this end, sterile mixtures of microbes are prepared, typically comprised of Clostridia, Bacteroides, Streptococcus, and Peptostreptococcus species. Petrof et al composed a stoolderived mixture of 33 strains of bacteria to "rePOOPulate" the gut and demonstrated efficacy with direct colonic administration in 2 patients, both of whom remained symptom free at 6 months. ${ }^{84,88}$ Similarly, Khanna et al reported the investigational compound SER-109, composed primarily of bacterial spores and administered as a pill to be promising as well, with a greater than $90 \%$ cure rate. ${ }^{89}$

In the end, the exact pathophysiology of how $C$. difficile infection occurs after antibiotic exposure may be less relevant than the composition and function of the gut microbiota that predisposes to this infection. Newer and more defined microbial ecosystems for treatment of $C$. difficile are becoming more prevalent and easier to administer, and they are certainly promising alternatives to conventional fecal microbiota transplantation or traditional antibiotic treatment for antibioticassociated diarrheal infections.

\section{Gut Microbiota in Malignancy}

Gastrointestinal cancers are a leading cause of cancer-related death globally with colon cancer being the second leading cause of cancer-related death in the United States. ${ }^{90}$ While genetic mutations in adenomatous polyposis coli (APC), p53, and KRAS have long been known to increase the risk of cancerous transformation, non-genetic factors, such as environmental and infectious causes, are being increasingly identified as carcinogenic. ${ }^{91}$ Recent conservative estimates have implicated microbial species in more than $15 \%$ of cancer cases, leading to at least 1.2 million cases per year of malignancy due to resident microbes. ${ }^{90}$ The GI microbiome is thought to increase carcinogenesis by inducing chronic inflammation through oxidative and nitrosative DNA damage, increasing cell proliferation, and producing mutagenic metabolites that affect DNA integrity. ${ }^{92,93}$ Additionally, the GI microbiome has also been implicated in altering the response to chemotherapeutic agents as well as immune surveillance mechanisms.

The well-studied causal relationship between a microbial agent and malignancy is the development of gastric cancer secondary to chronic infection with the gram-negative bacteria Helicobacter pylori, with 660,000 new cases of related gastric adenocarcinoma per year. ${ }^{90}$ However, not all colonized individuals go on to develop neoplasia, suggesting that the host response and the interaction between $H$. pylori and the rest of the GI microbiome also play key roles in carcinogenesis. H. pylori-negative individuals have highly diverse gastric microbiota, with increased Firmicutes, Bacteroidetes, and Actinobacteria, whereas $H$. pylori significantly reduces the diversity of the gastric microbiota, accounting for more than $90 \%$ of the detected bacteria in affected individuals. In addition, patients with $H$. pylori have increases in Proteobacteria, Spirochetes, and Acidobacteria. ${ }^{12}$ In individuals who go on to develop gastric cancer, Streptococcus mitis, S.parasanguinis, Lactobacillus, Veillonella, and Prevotella dominate the microbiome. ${ }^{94,95}$

The varying composition in the gastric microbiota alone does not explain the progression to gastric cancer, and further studies are needed to identify how the interactions between $H$. pylori and other members of the gastric microbiome promote malignant change following gastric atrophy in order to identify new therapeutic targets for chemoprevention.

Development of colorectal cancer (CRC) is related to both age and genetic predisposition, with cancer-associated mutations identified in the tumor-suppressor genes Apc, p53, KRAS, and catenin beta 1 (beta catenin). ${ }^{91}$ The colonic intestinal microbial composition is clearly implicated in tumorigenesis, because the incidence of tumors in the colon is 12 -fold higher than tumors of the small intestine, which has significantly fewer bacteria than the colon. ${ }^{96}$ Studies looking directly at the relationship between the gut microbiome and the pathogenesis of colorectal cancer have assessed either mucosa-associated bacteria or stool samples. There is an increase in Bacteroides species, whose toxin production increases inflammation, Coriobacteridaespecies, and Fusobacterium species. ${ }^{97-99} \mathrm{Wu}$ et al studied fecal samples from patients with CRC $(n=344)$ and healthy individuals $(\mathrm{n}=344)$ and showed increased Enterococcus and Streptococcus in CRC and increased butyrate-producing bacteria (Roseburia, Clostridium) in the controls. ${ }^{100}$ Similarly, work by Sobhaniet a indicated higher Bacteroides and Prevotella in CRC, whereas other researchers have observed higher proportions of Enterococcus, 
Escherichia, Shigella, B.fragilis, and Klebsiella with decreased butyrate-producing Roseburia. ${ }^{101,102}$ The findings from fecal samples were also confirmed by tissue sampling. Castellarin et al detected increased Fusobacterium nucleatum in cancerous tissues, with others detecting higher levels of Proteobacteria and Faecalibacterium in adenomas. Kostic et al confirmed increased Fusobacterium and Streptococcaceae in tumors. ${ }^{98,103-105}$

While the majority of studies have focused on relating CRC pathogenesis to a change in microbiota composition, research is also underway to assess the potential contributions of individual bacterial species to malignancy. For example, a recent study has revealed that Fusobacterium nucleatum can promote recruitment of tumor-infiltrating immune cells by creating an inflammatory environment conducive to the progression of colorectal neoplasia. ${ }^{104}$

The role of diet in the development of cancer is perhaps more important in the colon than any other part of the GI tract. The Western diet, rich in meat and fat, is known to be a risk factor in the development of CRC, whereas a fiber-rich diet has a protective effect ${ }^{106,107}$ by enhancing the production of shortchain fatty acids. As gut microbiota composition studies have shown, butyrate, produced by Faecalibacterium prausnitzii, Eubacterium and Roseburia, is anti-tumorigenic and is associated with decreased CRC. ${ }^{107}$ Lower levels of butyrateproducing bacteria are present both in meat-eating African Americans who are at an increased for CRC and in patients with lower fiber diets. ${ }^{108}$ Another area of growing interest pertaining to diet is hydrogen sulfide production by sulfitereducing bacteria.. The amount of genotoxic hydrogen sulfide produced depends on diet and the specific bacteria present in the gut. ${ }^{109}$

In addition to dietary modifications for prevention of CRC, using probiotics and antibiotics to modulate the intestinal microbiota has been studied in small scale using in vitro and animal models. Lactobacillus johnsonii and Lactobacillus casei suppress CRC tumor growth in patients and modulate the intestinal immune response. ${ }^{110,111}$ Antibiotics have long been used to eradicate $H$ pylori in the upper GI tract, and treatment is associated with a significant reduction in gastric cancer incidence up to 15 years after therapy. The use of antibiotics in CRC, however, has not been shown to have a beneficial effect, with recent studies from Boursi et al suggesting that long-term or frequent antibiotic exposure may negatively alter the gut microbiome by decreasing gut diversity and increasing the risk of CRC development (Boursi et al, abstract - Impact of Antibiotic Exposure on the Risk of Colorectal Cancer, 2014 ASCO Meeting).

Another growing area of interest is in modulating the gut microbiota to prevent toxicity associated with the chemotherapy and radiation used to treat cancer. A recent study identified the role of bacterial beta-glucuronidase in mediating GI toxicity of a common drug used for colon cancer CPT-11. ${ }^{12}$ Probiotics also appear to have a protective role in alleviating radiationrelated toxicity. ${ }^{113}$

In summary, while the alterations in gut microbiota between individuals who develop malignancies and those who do not have been well-described, studies exploring the exact pathophysiology of tumorigenesis have been limited. Early, small-scale work has shown that altering the microbiome can decrease the progression to cancer, thus making the microbiota an attractive target in chemoprevention. The use of either probiotics or more novel approaches such as fecal microbiota transplantation may be promising avenues to modulate the gut microbiota in individuals genetically susceptible to the development of GI-related cancers and may prove to be viable therapeutics as well.

\section{Gut Microbiota in Metabolic Disorders}

The rising incidence of obesity and associated conditions, including hypertension, hyperlipidemia, fatty liver, diabetes mellitus, and coronary artery disease, is an increasing public health concern in the United States and globally. Simply, weight gain is related to the imbalance between energy absorbed through food intake and energy expended through exercise and daily activities. However, emerging data suggest that the composition and diversity of bacteria in the gut may also increase the propensity for becoming obese.

Initial studies have focused on the gut microbiota composition. Early research identified an increase in the Firmicutes to Bacteroidetes ratio in both humans and mice genetically-predisposed to obesity but this finding does not appear to be robust given the lack of reproducibility. ${ }^{114,115}$ More recent metagenomic studies by Greenblum et al have demonstrated that there are differences between lean and obese microbiomes. ${ }^{116}$ The fluidity of the gut microbiome make reproducibility difficult and inconsistent results have made it difficult to form a consensus on the exact microbial footprint of obesity. This highlights two important aspects: first, the importance of controlling for patient characteristics, study design, and sequencing methodology among studies and 
second, the disadvantage of primarily focusing on gut microbiota composition alone.

While the composition of the gut microbiota in obesity remains in question, there are several lines of evidence supporting the role of gut microbiota in obesity. A functional analysis of gut microbial genes in twins concordant for obesity shows an increased capacity for carbohydrate processing by microbiota in the obese individuals. This suggests that differential metabolic capabilities of gut microbiota are important determinants of the host phenotype. ${ }^{117}$ Additionally, the transfer of gut bacteria from lean and obese individuals to germ-free mice is sufficient to transfer the lean or obese phenotype, providing further evidence for the role of the gut microbiota in obesity. ${ }^{118}$

Obesity-related conditions, such as diabetes and atherosclerosis, may also be driven by the gut microbiota. Obese individuals with insulin resistance have higher levels of circulating LPS and systemic inflammation implicating the gut microbiota (4-week high-fat diet has been shown to increase plasma LPS concentration two to three times). ${ }^{119}$ Metagenomewide association studies have identified reduced butyrate production by the microbiota to accompany obesity and subsequent insulin resistance, with both Qin et al and Karlsson et al reporting lower proportions of butyrate-producing Clostridiales, including Roseburia and Faecalibacterium prausnitzii, in obese individuals as well as increased proportions of Lactobacillus gasseri and Streptococcus mutans. ${ }^{120,121}$ Furthermore, the improvement in insulin sensitivity following fecal transplant from lean donors to obese individuals underlines the effect of the gut microbiota on the gut neurohormonal axis. Recent data on atherosclerosis have revealed that microbial metabolism of choline to trimethylamine, and subsequent conversion to trimethylamine- $\mathrm{N}$ oxide, is a significant risk factor for cardiovascular diseases. ${ }^{122}$

Modulation of the gut microbiota again represents an important opportunity for managing obesity. Traditional surgical approaches for treating obesity, such as Roux-en-Y gastric bypass, lead to alterations in gut microbiota in both human and mouse models. When this altered microbial community is transplanted into germ-free mice, improvements in metabolic parameters have been observed, suggesting that weight loss following surgery may be due in part to alteration in the gut microbiota. ${ }^{123}$ Specifically, there is an increase in F.prausnitzii in obese patients with type 2 diabetes after surgery, and levels of this organism are negatively correlated with inflammatory markers. This again suggests that this species may contribute to the improvement in insulin sensitivity following gastric bypass. ${ }^{124}$

Antibiotics reduce metabolic endotoxemia in mouse models of obesity and high fat-fed mice, as well as improve metabolic parameters such as glucose tolerance. ${ }^{125}$ However, with recent findings associating antibiotic use in early life with obesity as well as the rapidly increasing identification of multidrug resistant organisms, enthusiasm for using antibiotics to treat obesity remains low. ${ }^{126}$

We have mentioned previously the important role of both short- and long-term dietary patterns in determining the composition of the gut microbiome. Prebiotics and other dietary products known to modify the gut microbiome represent powerful tools to influence long-term change in obese patients. However, more targeted approaches are needed before such methods become widely used. Similarly, altering gut microbial community dynamics with probiotics, including Lactobacillus rhamnosus $G G$, have proven effective at controlling obesity as well. ${ }^{127,128}$

Lastly, transplantation of fecal microbiota is a potentially viable therapeutic option for the treatment of human obesity as it directly influences microbiota diversity and composition. An early study by Vriezeet al involved the transfer of fecal microbiota from lean donors to individuals with metabolic syndrome yielding improved insulin sensitivity and increased gut microbial diversity in the transplanted patients, with increased butyrate-producing intestinal bacteria at six weeks. ${ }^{129}$ With metagenomic analyses providing increasingly accurate depictions of the exact composition and functional role of the gut microbiome, targeted repletion with specific phyla or taxa of bacteria remains an attractive therapeutic intervention.

The data presented here underscore the role of gut microbiota in modulating host metabolic parameters, potentially influencing the development and progression of obesity and related disease states. As the composition of microbiota in obese and lean individuals becomes more defined, the role of individual bacterial species in the pathology of obesity will be revealed. Such information will allow for the development of targeted pre- and probiotic approaches to obesity treatment.

\section{Perspective}

Over the past decade we have made huge leaps in both science and technology as we try to elucidate the role of the microbiome in health and disease. The advances in Next- Generation sequencing and bioinformatics pipelines to handle Big Data, 
steady decline in cost of sequencing and the development of sophisticated animal models to study disease pathogenesis have led to significant observations not only in microbiome associated changes in different diseases but also in identifying their potential role in pathogenesis. Over the next decade as we may gain better understanding of host-microbial interactions in different disease states and specific pathways that lead to disease pathogenesis, we will be able to develop more specific microbiota targeted therapies such as purposeful alterations of diet, pre/pro/synbiotics, and fecal/defined microbial community transplant. Moreover, with the advent of newer synthetic biology tools, we can now envision use of genetically modified gut microbial community members for both diagnosis and delivery of therapeutics. Through better characterization of the microbiome in health and disease, research may eventually lead towards early diagnosis, better prognostication and intentionally modulating the makeup of the microbiome in favor of prevention rather than treatment.

\section{References}

1. Savage DC. Microbial ecology of the gastrointestinal tract. Annu Rev Microbiol. 1977;31:107-33.

2. Frank DN, St Amand AL, Feldman RA, Boedeker EC, Harpaz N, Pace NR. Molecular-phylogenetic characterization of microbial community imbalances in human inflammatory bowel diseases. Proc Natl Acad Sci U S A. 2007;104:13780-5.

3. Arpaia N, Rudensky AY. Microbial metabolites control gut inflammatory responses. Proc Natl Acad Sci U S A. 2014;111:2058-9.

4. Hollister EB, Gao C, Versalovic J. Compositional and functional features of the gastrointestinal microbiome and their effects on human health. Gastroenterology. 2014;146:1449-58.

5. Gill SR, Pop M, Deboy RT, Eckburg PB, Turnbaugh PJ, Samuel BS, et al. Metagenomic analysis of the human distal gut microbiome. Science. 2006;312:1355-9.

6. Pflughoeft KJ, Versalovic J. Human microbiome in health and disease. Annu Rev Pathol. 2012;7:99-122.

7. Eckburg PB, Bik EM, Bernstein CN, Purdom E, Dethlefsen L, Sargent M, et al. Diversity of the human intestinal microbial flora. Science. 2005;308:1635-8.

8. Aagaard K, Petrosino J, Keitel W, Watson M, Katancik J, Garcia $\mathrm{N}$, et al. The Human Microbiome Project strategy for comprehensive sampling of the human microbiome and why it matters. FASEB J. 2013;27:1012-22.

9. Morgan XC, Huttenhower C. Meta'omic analytic techniques for studying the intestinal microbiome. Gastroenterology. 2014;146:1437-48.

10. Arumugam M, Raes J, Pelletier E, Le Paslier D, Yamada T, Mende DR, et al. Enterotypes of the human gut microbiome. Nature. 2011;473:174-80.

11. Pei Z, Bini EJ, Yang L, Zhou M, Francois F, Blaser MJ. Bacterial biota in the human distal esophagus. Proc Natl Acad Sci U S A 2004;101:4250-5.

12. Bik EM, Eckburg PB, Gill SR, Nelson KE, Purdom EA, Francois F, et al. Molecular analysis of the bacterial microbiota in the human stomach. Proc Natl Acad Sci U S A. 2006;103:732-7.

13. Justesen T, Nielsen OH, Jacobsen IE, Lave J, Rasmussen SN. The normal cultivable microflora in upper jejunal fluid in healthy adults. Scand J Gastroenterol. 1984;19:279-82.

14. Relman DA. The human microbiome: ecosystem resilience and health. Nutr Rev. 2012;70:S2-9.

15. Lozupone CA, Stombaugh JI, Gordon JI, Jansson JK, Knight R. Diversity, stability and resilience of the human gut microbiota. Nature. 2012;489:220-30.

16. Dominguez-Bello MG, Blaser MJ, Ley RE, Knight R. Development of the human gastrointestinal microbiota and insights from high-throughput sequencing. Gastroenterology. 2011;140:1713-9.

17. Claesson MJ, Cusack S, O'Sullivan O, Greene-Diniz R, de Weerd H, Flannery E, et al. Composition, variability, and temporal stability of the intestinal microbiota of the elderly. Proc Natl Acad Sci U S A. 2011;108:4586-91.

18. LoCascio RG, Desai P, Sela DA, Weimer B, Mills DA. Broad conservation of milk utilization genes in Bifidobacterium longum subsp. infantis as revealed by comparative genomic hybridization. Appl Environ Microbiol. 2010;76:7373-81.

19. Lievin V, Peiffer I, Hudault S, Rochat F, Brassart D, Neeser JR, et al. Bifidobacterium strains from resident infant human gastrointestinal microflora exert antimicrobial activity. Gut. 2000;47:646-52.

20. De Filippo C, Cavalieri D, Di Paola M, Ramazzotti M, Poullet $\mathrm{JB}$, Massart S, et al. Impact of diet in shaping gut microbiota revealed by a comparative study in children from Europe and rural Africa. Proc Natl Acad Sci U S A. 2010;107:14691-6.

21. Sartor RB. Microbial influences in inflammatory bowel diseases. Gastroenterology. 2008;134:577-94.

22. Loftus EV, Jr. Clinical epidemiology of inflammatory bowel disease: Incidence, prevalence, and environmental influences. Gastroenterology. 2004;126:1504-17.

23. Swidsinski A, Ladhoff A, Pernthaler A, Swidsinski S, LoeningBaucke V, Ortner M, et al. Mucosal flora in inflammatory bowel disease. Gastroenterology. 2002;122:44-54.

24. Jostins L, Ripke S, Weersma RK, Duerr RH, McGovern DP, Hui KY, et al. Host-microbe interactions have shaped the genetic architecture of inflammatory bowel disease. Nature. 2012;491:119-24.

25. Flint HJ, Scott KP, Louis P and Duncan SH. The role of the gut microbiota in nutrition and health. Nat Rev Gastroenterol Hepatol. 2012;9:577-89.

26. Blaser MJ and Falkow S. What are the consequences of the disappearing human microbiota? Nat Rev Microbiol. 2009;7:887-94.

27. Pirzer U, Schonhaar A, Fleischer B, Hermann E, Meyer zum Buschenfelde KH. Reactivity of infiltrating T lymphocytes with microbial antigens in Crohn's disease. Lancet. 1991;338:1238-9.

28. Manichanh C, Rigottier-Gois L, Bonnaud E, Gloux K, Pelletier E, Frangeul L, et al. Reduced diversity of faecal microbiota in 
Crohn's disease revealed by a metagenomic approach. Gut. 2006;55:205-11.

29. Dicksved J, Halfvarson J, Rosenquist M, Jarnerot G, Tysk C, Apajalahti J, et al. Molecular analysis of the gut microbiota of identical twins with Crohn's disease. ISME J. 2008;2:716-27.

30. Kang S, Denman SE, Morrison M, Yu Z, Dore J, Leclerc M, et al. Dysbiosis of fecal microbiota in Crohn's disease patients as revealed by a custom phylogenetic microarray. Inflamm Bowel Dis. 2010;16:2034-42.

31. Darfeuille-Michaud A, Boudeau J, Bulois P, Neut C, Glasser AL, Barnich N, et al. High prevalence of adherent-invasive Escherichia coli associated with ileal mucosa in Crohn's disease. Gastroenterology. 2004;127:412-21.

32. Sokol H, Seksik P, Rigottier-Gois L, Lay C, Lepage P, Podglajen I, et al. Specificities of the fecal microbiota in inflammatory bowel disease. Inflamm Bowel Dis. 2006;12:106-11.

33. Ohkusa T, Okayasu I, Ogihara T, Morita K, Ogawa M, Sato N. Induction of experimental ulcerative colitis by Fusobacterium varium isolated from colonic mucosa of patients with ulcerative colitis. Gut. 2003;52:79-83.

34. Ohkusa T, Sato N, Ogihara T, Morita K, Ogawa M,Okayasu I. Fusobacterium varium localized in the colonic mucosa of patients with ulcerative colitis stimulates species-specific antibody. $J$ Gastroenterol Hepatol. 2002;17:849-53.

35. Ohkusa T, Yoshida T, Sato N, Watanabe S, Tajiri H, Okayasu I. Commensal bacteria can enter colonic epithelial cells and induce proinflammatory cytokine secretion: a possible pathogenic mechanism of ulcerative colitis. J Med Microbiol. 2009;58:535-45.

36. Sokol H, Pigneur B, Watterlot L, Lakhdari O, BermudezHumaran LG, Gratadoux JJ, et al. Faecalibacterium prausnitzii is an anti-inflammatory commensal bacterium identified by gut microbiota analysis of Crohn disease patients. Proc Natl Acad Sci U S A. 2008;105:16731-6.

37. Willing B, Halfvarson J, Dicksved J, Rosenquist M, Jarnerot G, Engstrand L, et al. Twin studies reveal specific imbalances in the mucosa-associated microbiota of patients with ileal Crohn's disease. Inflamm Bowel Dis. 2009;15:653-60.

38. Atarashi K, Tanoue T, Oshima K, Suda W, Nagano Y, Nishikawa $\mathrm{H}$, et al. Treg induction by a rationally selected mixture of Clostridia strains from the human microbiota. Nature. 2013;500:232-6.

39. Morgan XC, Tickle TL, Sokol H, Gevers D, Devaney KL, Ward DV, et al. Dysfunction of the intestinal microbiome in inflammatory bowel disease and treatment. Genome Biol. 2012;13: R79.

40. Sartor RB. Therapeutic manipulation of the enteric microflora in inflammatory bowel diseases: antibiotics, probiotics, and prebiotics. Gastroenterology. 2004;126:1620-33.

41. Kelly CR, Ihunnah C, Fischer M, Khoruts A, Surawicz C, Afzali A, et al. Fecal microbiota transplant for treatment of Clostridium difficile infection in immunocompromised patients. Am J Gastroenterol. 2014;109:1065-71.

42. Kunde S, Pham A, Bonczyk S, Crumb T, Duba M, Conrad H, Jr., et al. Safety, tolerability, and clinical response after fecal transplantation in children and young adults with ulcerative colitis. J Pediatr Gastroenterol Nutr. 2013;56:597-601.
43. Scaldaferri F, Gerardi V, Lopetuso LR, Del Zompo F, Mangiola F, Boskoski I, et al. Gut microbial flora, prebiotics, and probiotics in IBD: their current usage and utility. Biomed Res Int. 2013;2013:435268.

44. Welters CF, Heineman E, Thunnissen FB, van den Bogaard AE, Soeters PB, Baeten CG. Effect of dietary inulin supplementation on inflammation of pouch mucosa in patients with an ileal pouchanal anastomosis. Dis Colon Rectum. 2002;45:621-7.

45. Kashyap PC, Reigstad CS and Loftus EV, Jr. Role of diet and gut microbiota in management of inflammatory bowel disease in an Asian migrant. J Allergy Clin Immunol. 2013;132:250-e5.

46. Cui HH, Chen CL, Wang JD, Yang YJ, Cun Y, Wu JB, et al. Effects of probiotic on intestinal mucosa of patients with ulcerative colitis. World J Gastroenterol. 2004;10:1521-5.

47. Bibiloni R, Fedorak RN, Tannock GW, Madsen KL, Gionchetti $\mathrm{P}$, Campieri M, et al. VSL\#3 probiotic-mixture induces remission in patients with active ulcerative colitis. Am J Gastroenterol. 2005;100:1539-46.

48. Miele E, Pascarella F, Giannetti E, Quaglietta L, Baldassano RN and Staiano A. Effect of a probiotic preparation (VSL\#3) on induction and maintenance of remission in children with ulcerative colitis. Am J Gastroenterol. 2009;104:437-43.

49. Kruis W, Fric P, Pokrotnieks J, Lukas M, Fixa B, Kascak M, et al. Maintaining remission of ulcerative colitis with the probiotic Escherichia coli Nissle 1917 is as effective as with standard mesalazine. Gut. 2004;53:1617-23.

50. Gionchetti P, Rizzello F, Helwig U, Venturi A, Lammers KM, Brigidi $\mathrm{P}$, et al. Prophylaxis of pouchitis onset with probiotic therapy: a double-blind, placebo-controlled trial. Gastroenterology. 2003;124:1202-9.

51. Schultz M, Timmer A, Herfarth HH, Sartor RB, Vanderhoof JA and Rath HC. Lactobacillus GG in inducing and maintaining remission of Crohn's disease. BMC Gastroenterol. 2004;4:5.

52. Bousvaros A, Guandalini S, Baldassano RN, Botelho C, Evans J, Ferry GD, et al. A randomized, double-blind trial of Lactobacillus GG versus placebo in addition to standard maintenance therapy for children with Crohn's disease. Inflamm Bowel Dis. 2005;11:833-9.

53. Rolfe VE, Fortun PJ, Hawkey CJ and Bath-Hextall F. Probiotics for maintenance of remission in Crohn's disease. Cochrane Database Syst Rev. 2006:CD004826.

54. Hungin AP, Chang L, Locke GR, Dennis EH and Barghout V. Irritable bowel syndrome in the United States: prevalence, symptom patterns and impact. Aliment Pharmacol Ther. 2005;21:1365-75.

55. Longstreth GF, Thompson WG, Chey WD, Houghton LA, Mearin F and Spiller RC. Functional bowel disorders. Gastroenterology. 2006;130:1480-91.

56. Mayer EA, Savidge T and Shulman RJ. Brain-gut microbiome interactions and functional bowel disorders. Gastroenterology. 2014;146:1500-12.

57. Matto J, Maunuksela L, Kajander K, Palva A, Korpela R, Kassinen A, et al. Composition and temporal stability of gastrointestinal microbiota in irritable bowel syndrome-a longitudinal study in IBS and control subjects. FEMS Immunol Med Microbiol. 2005;43:213-22.

58. Balsari A, Ceccarelli A, Dubini F, Fesce E and Poli G. The fecal 
microbial population in the irritable bowel syndrome. Microbiologica. 1982;5:185-94.

59. Malinen E, Rinttila T, Kajander K, Matto J, Kassinen A, Krogius $\mathrm{L}$, et al. Analysis of the fecal microbiota of irritable bowel syndrome patients and healthy controls with real-time PCR. Am J Gastroenterol. 2005;100:373-82.

60. Wang ZK and Yang YS. Upper gastrointestinal microbiota and digestive diseases. World J Gastroenterol. 2013;19:1541-50.

61. Simren M, Barbara G, Flint HJ, Spiegel BM, Spiller RC, Vanner $\mathrm{S}$, et al. Intestinal microbiota in functional bowel disorders: a Rome foundation report. Gut. 2013;62:159-76.

62. Bailey MT and Coe CL. Maternal separation disrupts the integrity of the intestinal microflora in infant rhesus monkeys. Dev Psychobiol. 1999;35:146-55.

63. Brint EK, MacSharry J, Fanning A, Shanahan F and Quigley EM. Differential expression of toll-like receptors in patients with irritable bowel syndrome. Am J Gastroenterol. 2011;106:329-36.

64. Rhee SH, Pothoulakis C and Mayer EA. Principles and clinical implications of the brain-gut-enteric microbiota axis. Nat Rev Gastroenterol Hepatol. 2009;6:306-14.

65. Hughes PA, Zola H, Penttila IA, Blackshaw LA, Andrews JM and Krumbiegel D. Immune activation in irritable bowel syndrome: can neuroimmune interactions explain symptoms? Am J Gastroenterol. 2013;108:1066-74.

66. Halmos EP, Power VA, Shepherd SJ, Gibson PR and Muir JG. A diet low in FODMAPs reduces symptoms of irritable bowel syndrome. Gastroenterology. 2014;146:67-75 e5.

67. Silk DB, Davis A, Vulevic J, Tzortzis G and Gibson GR. Clinical trial: the effects of a trans-galactooligosaccharide prebiotic on faecal microbiota and symptoms in irritable bowel syndrome. Aliment Pharmacol Ther. 2009;29:508-18.

68. Ortiz-Lucas M, Tobias A, Saz P and Sebastian JJ. Effect of probiotic species on irritable bowel syndrome symptoms: A bring up to date meta-analysis. Rev Esp Enferm Dig. 2013;105:19-36.

69. Horvath A, Dziechciarz P and Szajewska H. Meta-analysis: Lactobacillus rhamnosus GG for abdominal pain-related functional gastrointestinal disorders in childhood. Aliment Pharmacol Ther. 2011;33:1302-10.

70. Xu D, Gao J, Gillilland M, 3rd, Wu X, Song I, Kao JY, et al. Rifaximin alters intestinal bacteria and prevents stress-induced gut inflammation and visceral hyperalgesia in rats. Gastroenterology. 2014;146:484-96.

71. Britton RA and Young VB. Role of the intestinal microbiota in resistance to colonization by Clostridium difficile. Gastroenterology. 2014;146:1547-53.

72. Dubberke E. Clostridium difficile infection: the scope of the problem. J Hosp Med. 2012;7:S1-4.

73. Thompson CM, Jr., Gilligan PH, Fisher MC and Long SS. Clostridium difficile cytotoxin in a pediatric population. Am J Dis Child. 1983;137:271-4.

74. Bohnhoff M, Drake BL and Miller CP. Effect of streptomycin on susceptibility of intestinal tract to experimental Salmonella infection. Proc Soc Exp Biol Med. 1954;86:132-7.

75. Chen X, Katchar K, Goldsmith JD, Nanthakumar N, Cheknis A, Gerding DN, et al. A mouse model of Clostridium difficile- associated disease. Gastroenterology. 2008;135:1984-92.

76. Lawley TD, Clare S, Walker AW, Stares MD, Connor TR, Raisen $\mathrm{C}$, et al. Targeted restoration of the intestinal microbiota with a simple, defined bacteriotherapy resolves relapsing Clostridium difficile disease in mice. PLoS Pathog. 2012;8:e1002995.

77. Britton RA and Young VB. Interaction between the intestinal microbiota and host in Clostridium difficile colonization resistance. Trends Microbiol. 2012;20:313-9.

78. Wilson KH. Efficiency of various bile salt preparations for stimulation of Clostridium difficile spore germination. J Clin Microbiol. 1983;18:1017-9.

79. Wilson KH and Perini F. Role of competition for nutrients in suppression of Clostridium difficile by the colonic microflora. Infect Immun. 1988;56:2610-4.

80. Peterfreund GL, Vandivier LE, Sinha R, Marozsan AJ, Olson WC, Zhu J, et al. Succession in the gut microbiome following antibiotic and antibody therapies for Clostridium difficile. PLoS One. 2012;7:e46966.

81. Cohen SH, Gerding DN, Johnson S, Kelly CP, Loo VG, McDonald LC, et al. Clinical practice guidelines for Clostridium difficile infection in adults: 2010 update by the society for healthcare epidemiology of America (SHEA) and the infectious diseases society of America (IDSA). Infect Control Hosp Epidemiol. 2010;31:431-55.

82. Venugopal AA and Johnson S. Fidaxomicin: a novel macrocyclic antibiotic approved for treatment of Clostridium difficile infection. Clin Infect Dis. 2012;54:568-74.

83. Banerjee P, Merkel GJ and Bhunia AK. Lactobacillus delbrueckii ssp. bulgaricus B-30892 can inhibit cytotoxic effects and adhesion of pathogenic Clostridium difficile to Caco-2 cells. Gut Pathog. 2009;1:8.

84. Petrof EO, Gloor GB, Vanner SJ, Weese SJ, Carter D, Daigneault $\mathrm{MC}$, et al. Stool substitute transplant therapy for the eradication of Clostridium difficile infection: 'RePOOPulating' the gut. Microbiome. 2013;1:3.

85. Tvede M and Rask-Madsen J. Bacteriotherapy for chronic relapsing Clostridium difficile diarrhoea in six patients. Lancet. 1989;1:1156-60.

86. van Nood E, Vrieze A, Nieuwdorp M, Fuentes S, Zoetendal EG, de Vos WM, et al. Duodenal infusion of donor feces for recurrent Clostridium difficile. $N$ Engl J Med. 2013;368:407-15.

87. Khoruts A, Dicksved J, Jansson JK and Sadowsky MJ. Changes in the composition of the human fecal microbiome after bacteriotherapy for recurrent Clostridium difficile-associated diarrhea. J Clin Gastroenterol. 2010;44:354-60.

88. Petrof EO and Khoruts A. From stool transplants to nextgeneration microbiota therapeutics. Gastroenterology. 2014; 146:1573-82.

89. Khanna S and Pardi DS. Clostridium difficile infection: management strategies for a difficult disease. Therap $A d v$ Gastroenterol. 2014;7:72-86.

90. de Martel C, Ferlay J, Franceschi S, Vignat J, Bray F, Forman $\mathrm{D}$, et al. Global burden of cancers attributable to infections in 2008: a review and synthetic analysis. Lancet Oncol. 2012;13:607-15.

91. Abreu MT and Peek RM, Jr. Gastrointestinal malignancy and 
the microbiome. Gastroenterology. 2014;146:1534-46.

92. Cho I and Blaser MJ. The human microbiome: at the interface of health and disease. Nat Rev Genet. 2012;13:260-70.

93. Plottel CS and Blaser MJ. Microbiome and malignancy. Cell Host Microbe. 2011;10:324-35.

94. Maldonado-Contreras A, Goldfarb KC, Godoy-Vitorino F, Karaoz U, Contreras M, Blaser MJ, et al. Structure of the human gastric bacterial community in relation to Helicobacter pylori status. ISME J. 2011;5:574-9.

95. Yang I, Nell S and Suerbaum S. Survival in hostile territory: the microbiota of the stomach. FEMS Microbiol Rev. 2013;37:736-61.

96. Jemal A, Siegel R, Ward E, Hao Y, Xu J and Thun MJ. Cancer statistics, 2009. CA Cancer J Clin. 2009;59:225-49.

97. Wu N, Yang X, Zhang R, Li J, Xiao X, Hu Y, et al. Dysbiosis signature of fecal microbiota in colorectal cancer patients. Microb Ecol. 2013;66:462-70.

98. Castellarin M, Warren RL, Freeman JD, Dreolini L, Krzywinski M, Strauss J, et al. Fusobacterium nucleatum infection is prevalent in human colorectal carcinoma. Genome Res. 2012;22:299-306.

99. Weir TL, Manter DK, Sheflin AM, Barnett BA, Heuberger AL and Ryan EP. Stool microbiome and metabolome differences between colorectal cancer patients and healthy adults. PLoS One. 2013;8:e70803.

100. Wu S, Rhee KJ, Albesiano E, Rabizadeh S, Wu X, Yen HR, et al. A human colonic commensal promotes colon tumorigenesis via activation of $\mathrm{T}$ helper type $17 \mathrm{~T}$ cell responses. Nat Med. 2009;15:1016-22.

101. Sobhani I, Tap J, Roudot-Thoraval F, Roperch JP, Letulle S, Langella P, et al. Microbial dysbiosis in colorectal cancer (CRC) patients. PLoS One. 2011;6:e16393.

102. Grivennikov SI, Wang K, Mucida D, Stewart CA, Schnabl B, Jauch D, et al. Adenoma-linked barrier defects and microbial products drive IL-23/IL-17-mediated tumour growth. Nature. 2012;491:254-8.

103. Shen XJ, Rawls JF, Randall T, Burcal L, Mpande CN, Jenkins $\mathrm{N}$, et al. Molecular characterization of mucosal adherent bacteria and associations with colorectal adenomas. Gut Microbes. 2010;1:138-47.

104. Kostic AD, Chun E, Robertson L, Glickman JN, Gallini CA, Michaud M, et al. Fusobacterium nucleatum potentiates intestinal tumorigenesis and modulates the tumor-immune microenvironment. Cell Host Microbe. 2013;14:207-15.

105. Kostic AD, Gevers D, Pedamallu CS, Michaud M, Duke F, Earl $\mathrm{AM}$, et al. Genomic analysis identifies association of Fusobacterium with colorectal carcinoma. Genome Res. 2012;22:292-8.

106. Vargas AJ and Thompson PA. Diet and nutrient factors in colorectal cancer risk. Nutr Clin Pract. 2012;27:613-23.

107. O'Keefe SJ. Nutrition and colonic health: the critical role of the microbiota. Curr Opin Gastroenterol. 2008;24:51-8.

108. Ou J, Carbonero F, Zoetendal EG, DeLany JP, Wang M, Newton $\mathrm{K}$, et al. Diet, microbiota, and microbial metabolites in colon cancer risk in rural Africans and African Americans. Am J Clin Nutr. 2013;98:111-20.

109. Attene-Ramos MS, Wagner ED, Plewa MJ and Gaskins HR.
Evidence that hydrogen sulfide is a genotoxic agent. Mol Cancer Res. 2006;4:9-14.

110. Gianotti L, Morelli L, Galbiati F, Rocchetti S, Coppola S, Beneduce $\mathrm{A}$, et al. A randomized double-blind trial on perioperative administration of probiotics in colorectal cancer patients. World J Gastroenterol. 2010;16:167-75.

111. Ishikawa H, Akedo I, Otani T, Suzuki T, Nakamura T, Takeyama I, et al. Randomized trial of dietary fiber and Lactobacillus casei administration for prevention of colorectal tumors. Int J Cancer. 2005;116:762-7.

112. Wallace BD, Wang H, Lane KT, Scott JE, Orans J, Koo JS, et al. Alleviating cancer drug toxicity by inhibiting a bacterial enzyme. Science. 2010;330:831-5.

113. Ciorba MA, Riehl TE, Rao MS, Moon C, Ee X, Nava GM, et al. Lactobacillus probiotic protects intestinal epithelium from radiation injury in a TLR-2/cyclo-oxygenase-2-dependent manner. Gut. 2012;61:829-38.

114. Duncan SH, Lobley GE, Holtrop G, Ince J, Johnstone AM, Louis $\mathrm{P}$, et al. Human colonic microbiota associated with diet, obesity and weight loss. Int J Obes (Lond). 2008;32:1720-4.

115. Schwiertz A, Taras D, Schafer K, Beijer S, Bos NA, Donus C, et al. Microbiota and SCFA in lean and overweight healthy subjects. Obesity (Silver Spring). 2010;18:190-5.

116. Greenblum S, Turnbaugh PJ and Borenstein E. Metagenomic systems biology of the human gut microbiome reveals topological shifts associated with obesity and inflammatory bowel disease. Proc Natl Acad Sci U S A. 2012;109:594-9.

117. Turnbaugh PJ, Hamady M, Yatsunenko T, Cantarel BL, Duncan A, Ley RE, et al. A core gut microbiome in obese and lean twins. Nature. 2009;457:480-4.

118. Ridaura VK, Faith JJ, Rey FE, Cheng J, Duncan AE, Kau AL, et al. Gut microbiota from twins discordant for obesity modulate metabolism in mice. Science. 2013;341:1241214.

119. Cani PD, Amar J, Iglesias MA, Poggi M, Knauf C, Bastelica D, et al. Metabolic endotoxemia initiates obesity and insulin resistance. Diabetes. 2007;56:1761-72.

120. Qin J, Li Y, Cai Z, Li S, Zhu J, Zhang F, et al. A metagenomewide association study of gut microbiota in type 2 diabetes. Nature. 2012;490:55-60.

121. Karlsson FH, Tremaroli V, Nookaew I, Bergstrom G, Behre CJ, Fagerberg B, et al. Gut metagenome in European women with normal, impaired and diabetic glucose control. Nature. 2013;498:99-103.

122. Koeth RA, Wang Z, Levison BS, Buffa JA, Org E, Sheehy BT, et al. Intestinal microbiota metabolism of L-carnitine, a nutrient in red meat, promotes atherosclerosis. Nat Med. 2013;19:57685.

123. Liou AP, Paziuk M, Luevano JM, Jr., Machineni S, Turnbaugh PJ,Kaplan LM. Conserved shifts in the gut microbiota due to gastric bypass reduce host weight and adiposity. Sci Transl Med. 2013;5:178ra41.

124. Karlsson F, Tremaroli V, Nielsen J and Backhed F. Assessing the human gut microbiota in metabolic diseases. Diabetes. 2013;62:3341-9.

125. Cani PD, Bibiloni R, Knauf C, Waget A, Neyrinck AM, Delzenne $\mathrm{NM}$, et al. Changes in gut microbiota control metabolic endotoxemia-induced inflammation in high-fat diet-induced 
obesity and diabetes in mice. Diabetes. 2008;57:1470-81.

126. Bailey LC, Forrest CB, Zhang P, Richards TM, Livshits A,DeRusso PA. Association of Antibiotics in Infancy With Early Childhood Obesity. JAMA Pediatr. 2014:168:1063-9.

127. DiBaise JK, Zhang H, Crowell MD, Krajmalnik-Brown R,
Decker GA and Rittmann BE. Gut microbiota and its possible relationship with obesity. Mayo Clin Proc. 2008;83:460-9.

128. Moreno-Indias I, Cardona F, Tinahones FJ and Queipo-Ortuno MI. Impact of the gut microbiota on the development of obesity and type 2 diabetes mellitus. Front Microbiol. 2014;5:190.

129. Vrieze A, de Groot PF, Kootte RS, Knaapen M, van Nood E and Nieuwdorp M. Fecal transplant: a safe and sustainable clinical therapy for restoring intestinal microbial balance in human disease? Best Pract Res Clin Gastroenterol. 2013;27:127-37. 